\title{
COMPARATIVE STUDIES OF
}

\author{
SUBSTITUTION REACTIONS OF \\ RHENIUM (I) DICARBONYL-NITROSYL \\ AND TRICARBONYL COMPLEXES IN \\ AQUEOUS MEDIA
}

N. Marti, B. Spingler, F. Breher and R. Schibli 


\section{Table of Content}

NMR spectra of complex 3: S3

NMR spectra of complex 7: S4

NMR spectra of complex 4: S5

NMR spectra of complex 8: S6

NMR spectra of complex 5: S7

NMR spectra of complex 9: S8

NMR spectra of complex 6: S9

NMR spectra of complex 10: .S10 
NMR spectra of complex 3:

Pulse Sequence: s2pu

Solvent: D2O

Ambient temperature

GEMINI-300BB 'pss116'

Relax. delay 1.000 sec

Pulse 45.0 degrees

Acq. fime $1.998 \mathrm{sec}$

Width $4799.8 \mathrm{H}$

576 tepetitions
OBSERVE HI. 299.9866985 MHL

OBSERVE H1, 299.98

line broadening $1.0 \mathrm{~Hz}$

FT size 32768

Total time $1 \mathrm{hc}, 23 \mathrm{~min}, 5 \mathrm{sec}$

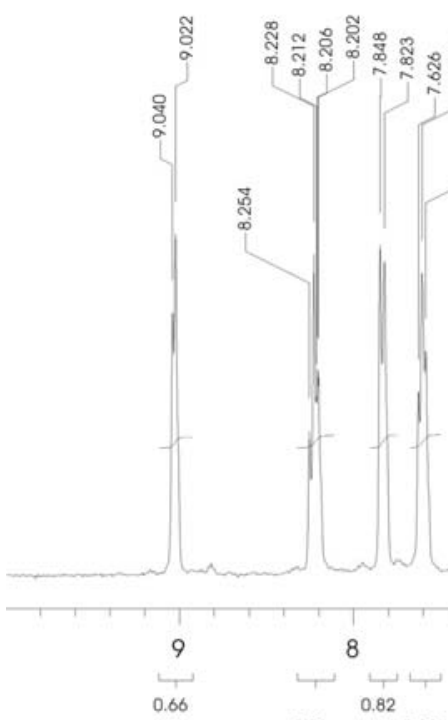

Pulse Sequence: s2pul

$0.79 \quad 0.82$

Solvent: DMSO

Ambient temperature

GEMINI-3008B 'pss116"

Relax, delay $1.000 \mathrm{sec}$

Pulse 45.0 degrees

Acq, time $1.815 \mathrm{sec}$

Widh $18858.2 \mathrm{~Hz}$

OBSERVE C13.75.4328943 MHE

DECOUPLE HI, 299.9927494 MHZ

Power $40 \mathrm{~dB}$

on during acquistion

off during delay

WALIZ-16 modulated

DATA PROCESSING

Line broadening $1.0 \mathrm{~Hz}$

FT size 131072

Total time $93865 \mathrm{hr}, 37 \mathrm{~min}, 36 \mathrm{sec}$

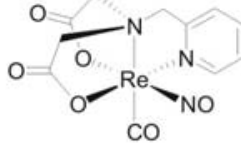

3
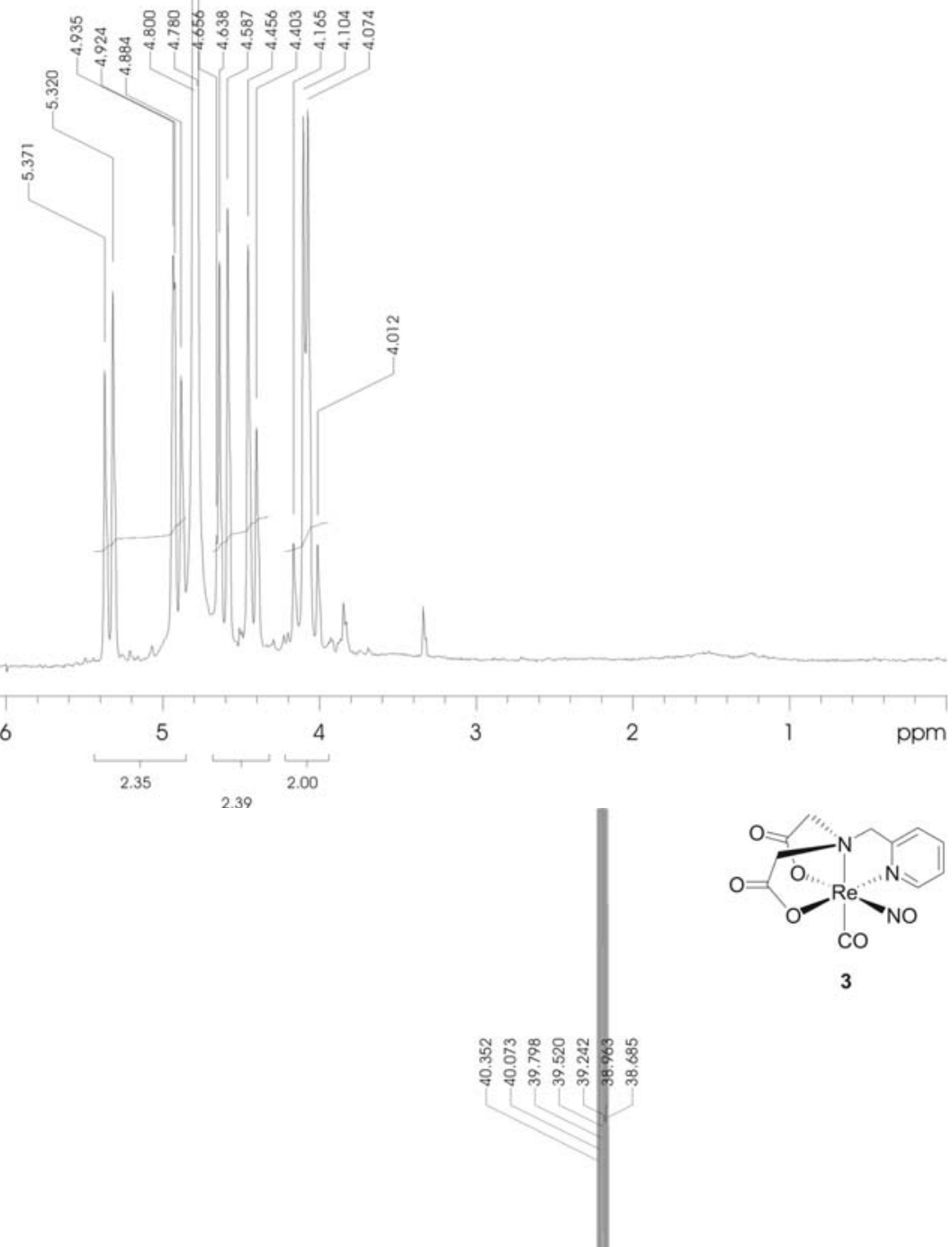

3

ㅊำ สู่

8

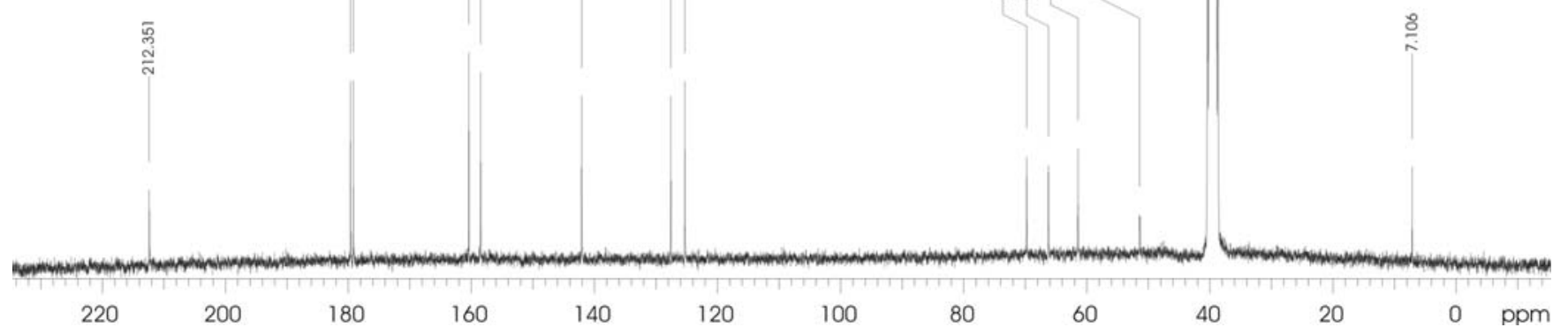


NMR spectra of complex 7:

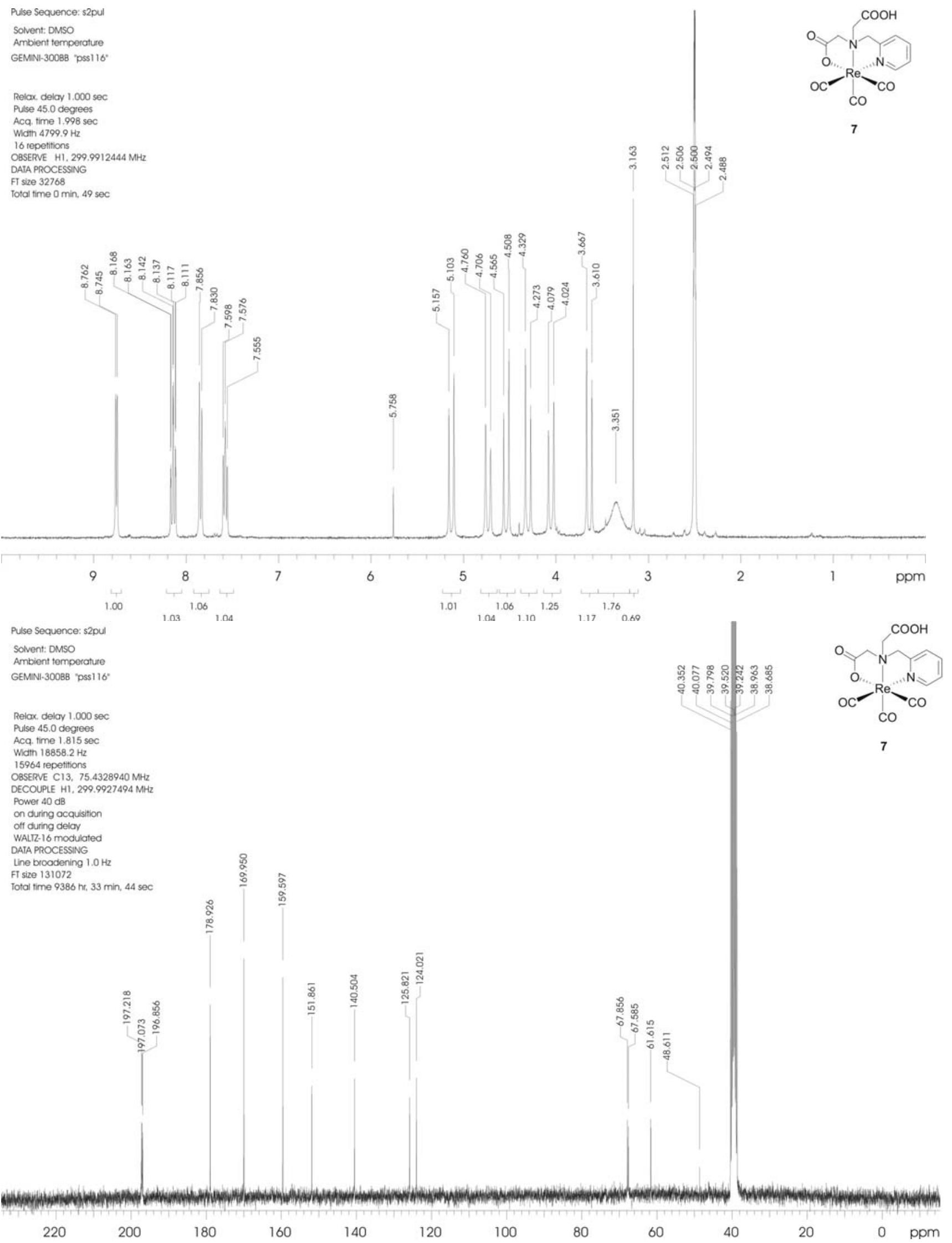


NMR spectra of complex 4:

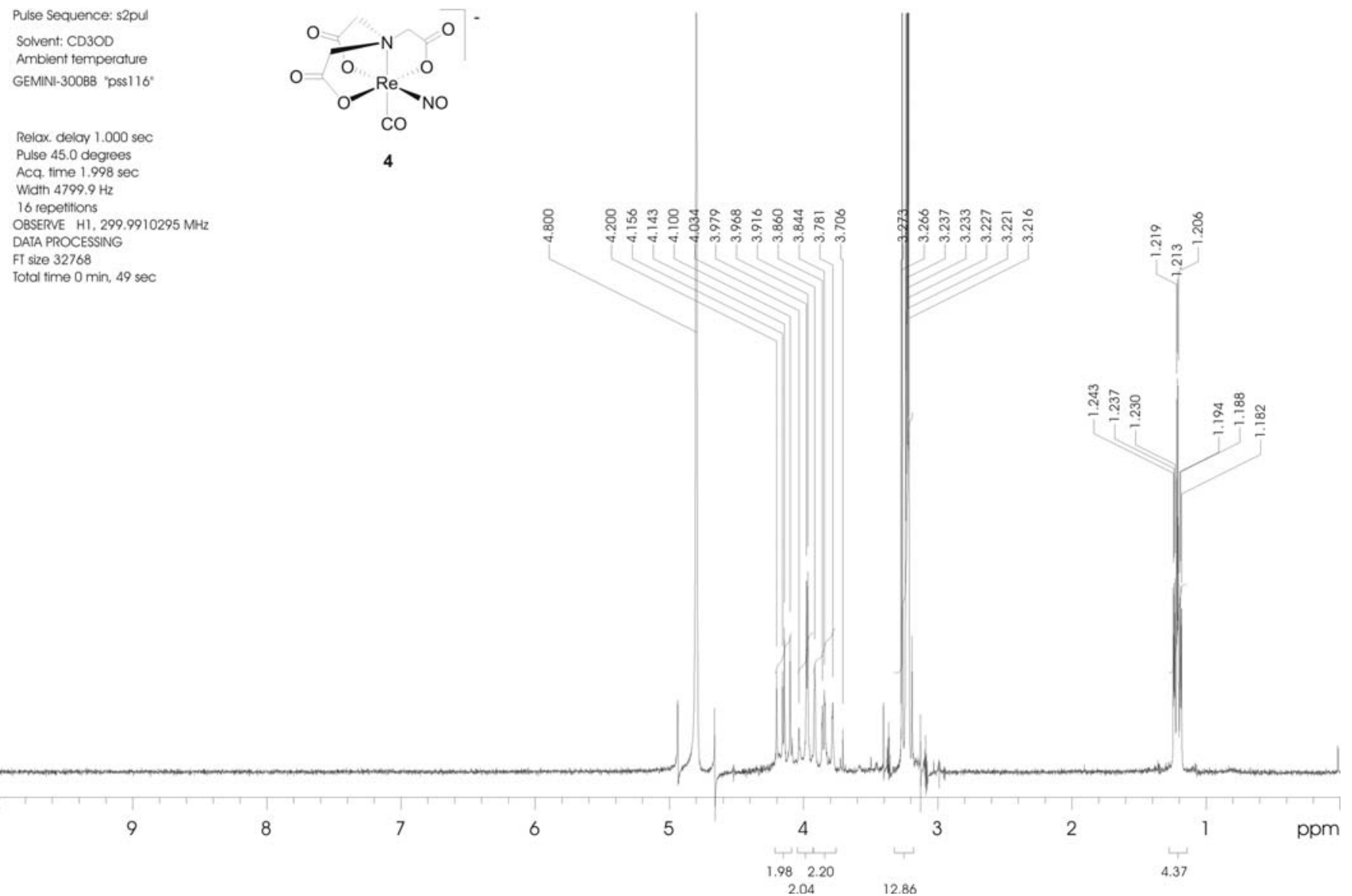


NMR spectra of complex 8:

Pulse Sequence: s2pul

Solvent: $C D 3 O D$

Temp. $23.0 \mathrm{C} / 296.1 \mathrm{~K}$

GEMINI-3008B "pss116"

Relax. delay $1.000 \mathrm{sec}$

Pulse 45.0 degrees

Acq, time $1.998 \mathrm{sec}$

Width $4799.9 \mathrm{~Hz}$

OBSEPVE HI, 299.9910169 MHL

DATA PROCESSING

FT size 32768

Total time $0 \mathrm{~min}, 49 \mathrm{sec}$

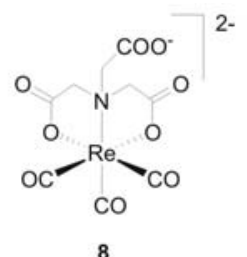

8

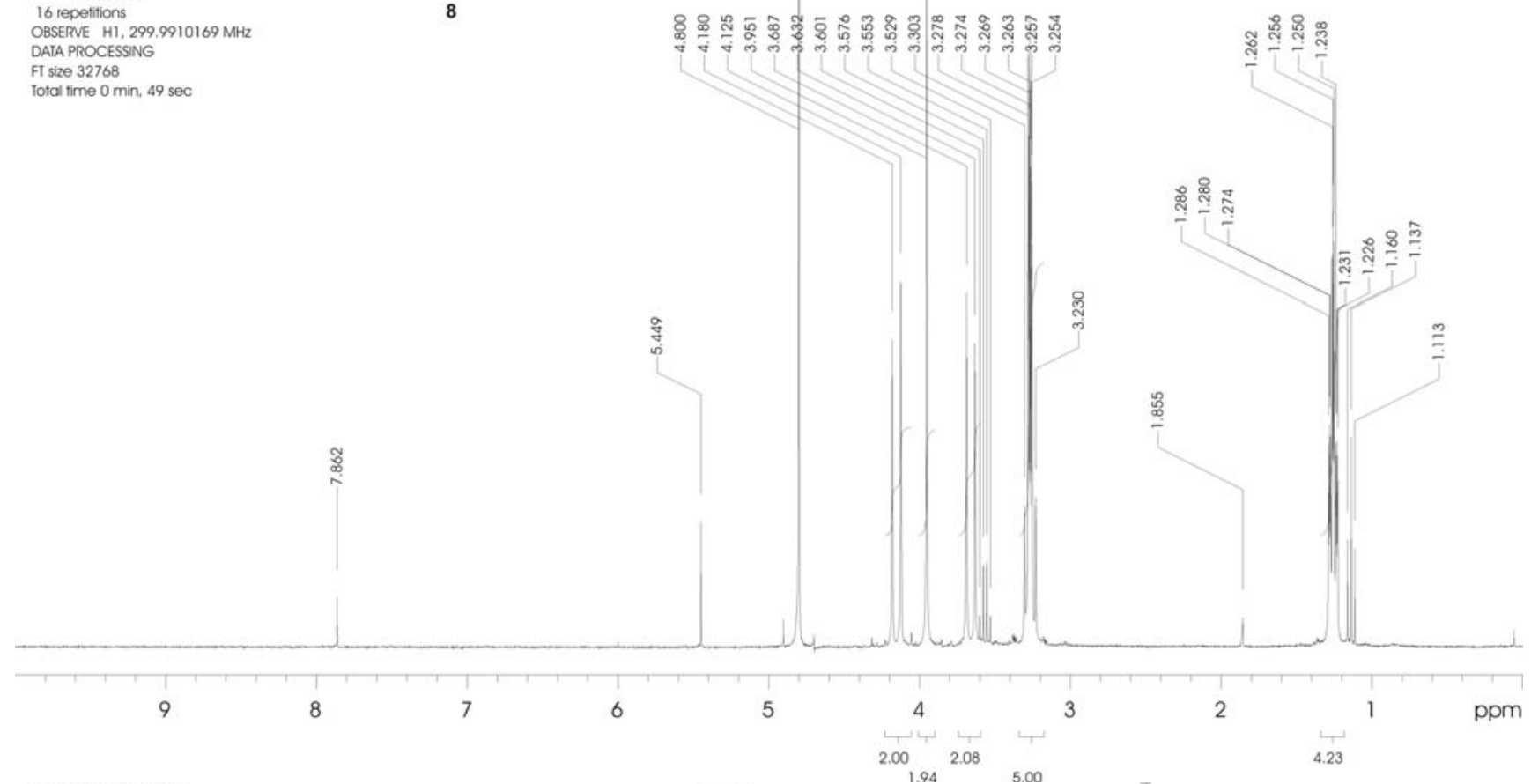

Pulse Sequence: s2pul

Solvent: CD3OD

Temp. $23.0 \mathrm{C} / 296.1 \mathrm{~K}$

GEMINI-3008B "pss116"

Relax. delay $1.000 \mathrm{sec}$

Pulse 45.0 degrees

Acq. time $1.815 \mathrm{sec}$

Width $18858.2 \mathrm{~Hz}$

65816 repetitions

OBSERVE C13, 75.4326945 MUt

DECOUPLE HI, $299.9925064 \mathrm{MHz}$

Power $40 \mathrm{~dB}$

on during acquisition

off during delay

WALIZ-16 modulated

DATA PROCESSING

Line broadening $1.0 \mathrm{~Hz}$

FT size 131072

Totol time $9386 \mathrm{he}, 34 \mathrm{~min}, 0 \mathrm{sec}$
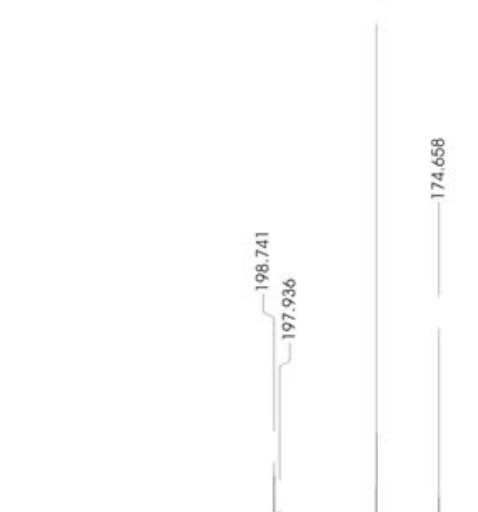

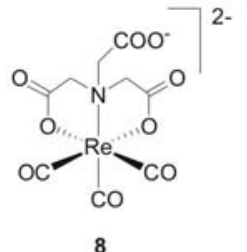

8

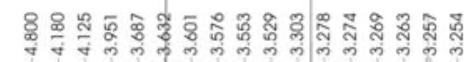

$1.94 \quad 5.00$

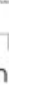


NMR spectra of complex 5:

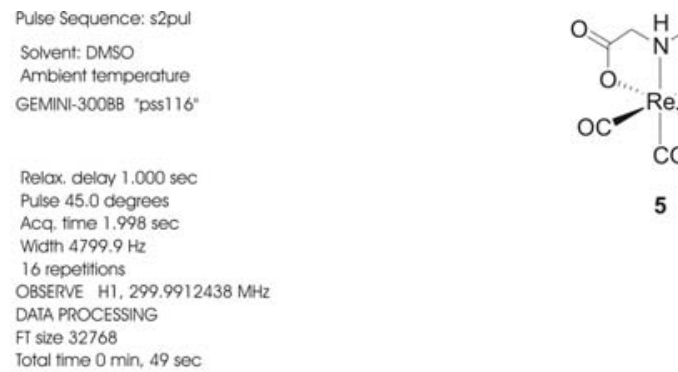

5

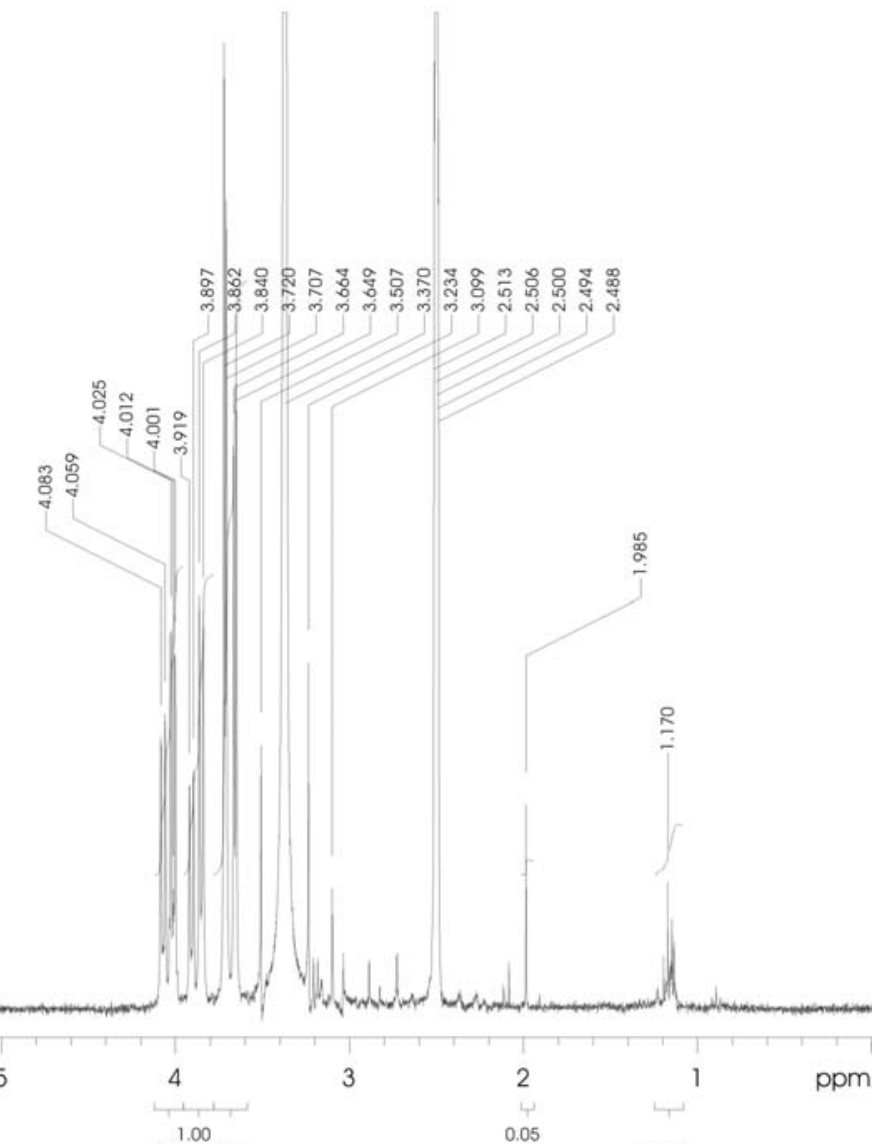

Pulse Sequence: 52pul

Solvent: DMSO

Ambient temperature

GEMNN-30088 'pss 116 '

$103 \quad 1.98$

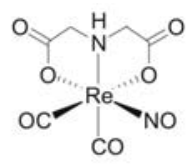

0.17

Relcx. delay $1.000 \mathrm{sec}$

Pulse 45.0 degrees

Acq. fime $1.815 \mathrm{sec}$

Wath $18858.2 \mathrm{~Hz}$

O

DECOUPLE H1, 299.9927494 MHz

Power $40 \mathrm{~dB}$

on during acquisition

off duing delay

Wallz-16 modulated

DATA PROCESSING

FT size 131072

Total time $9380 \mathrm{ht}, 33 \mathrm{~min}, 44 \mathrm{sec}$

5

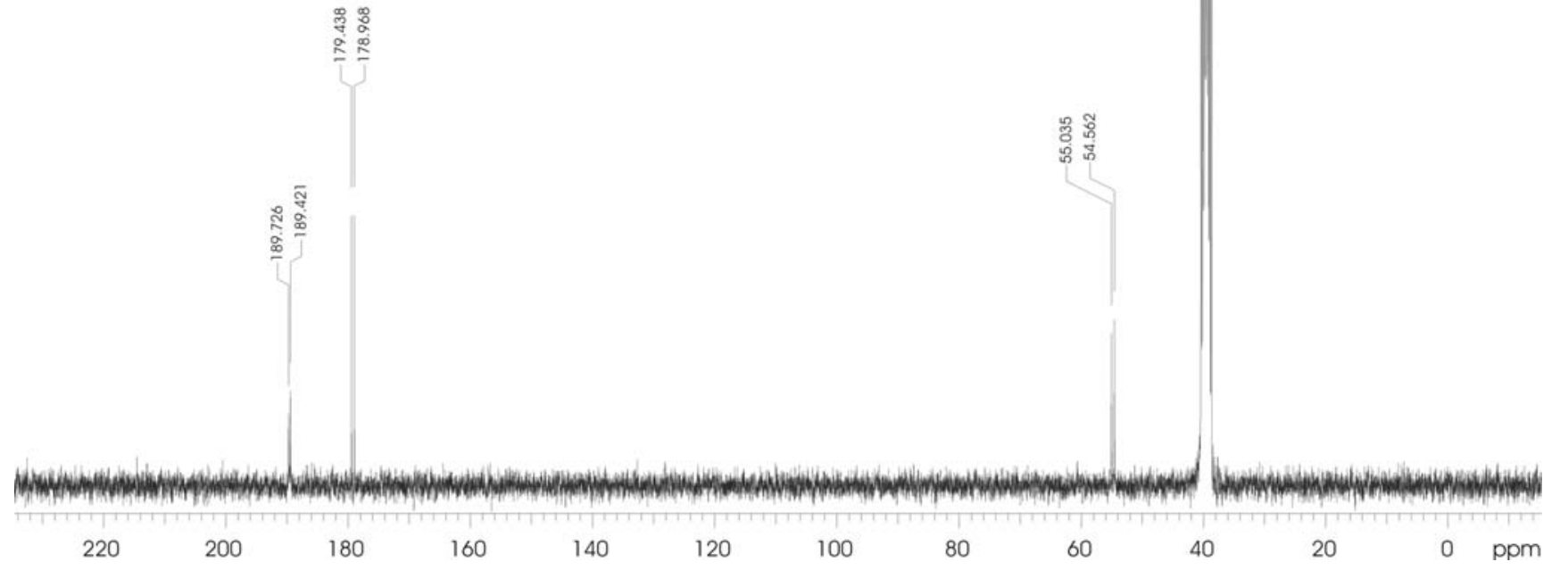


NMR spectra of complex 9:

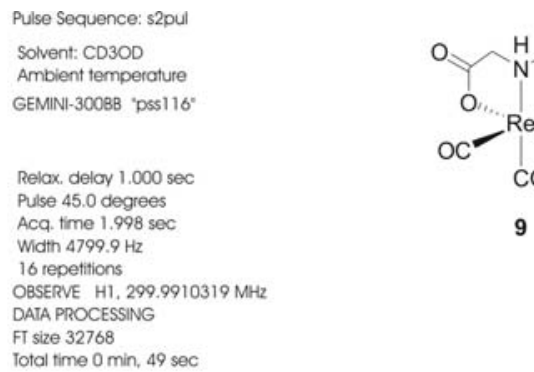

9

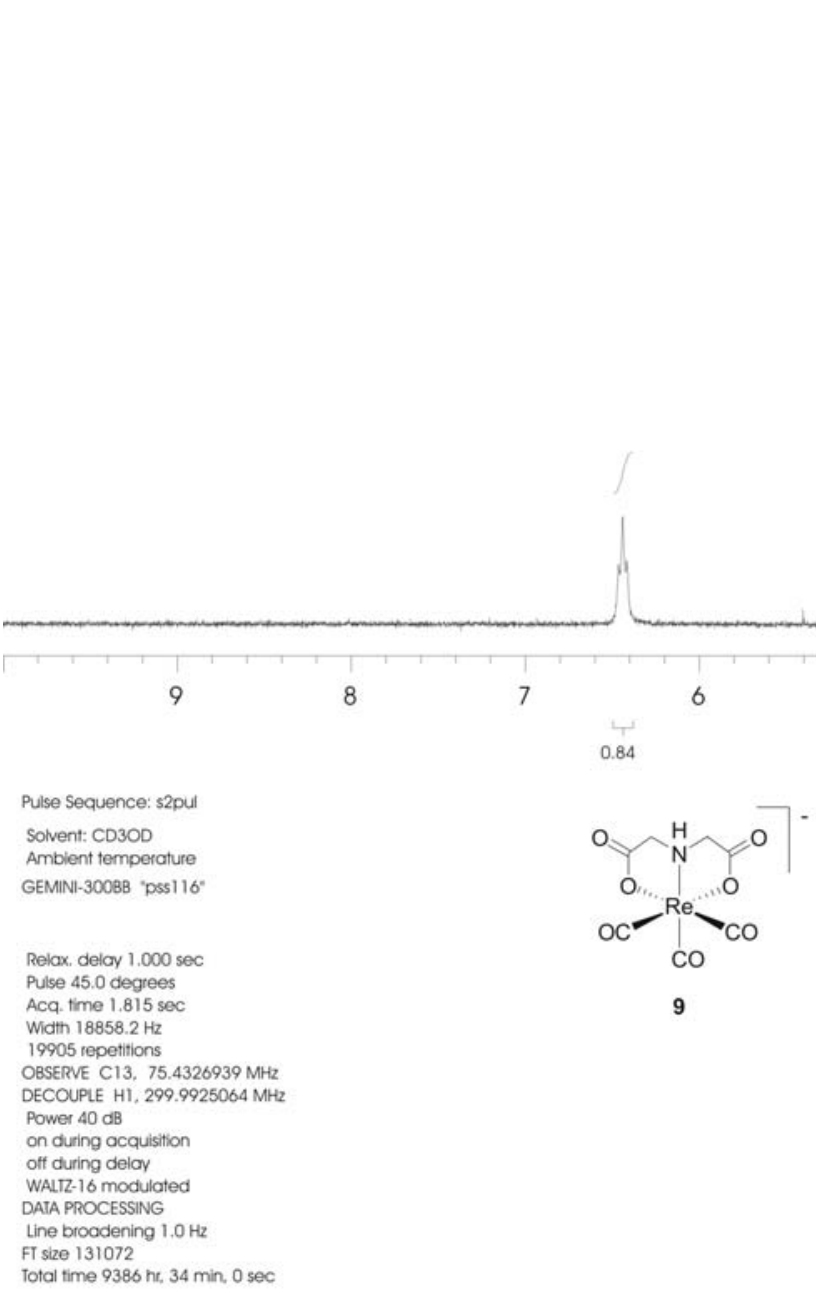

fime $9386 \mathrm{ht}, 34 \mathrm{~min}, 0 \mathrm{sec}$

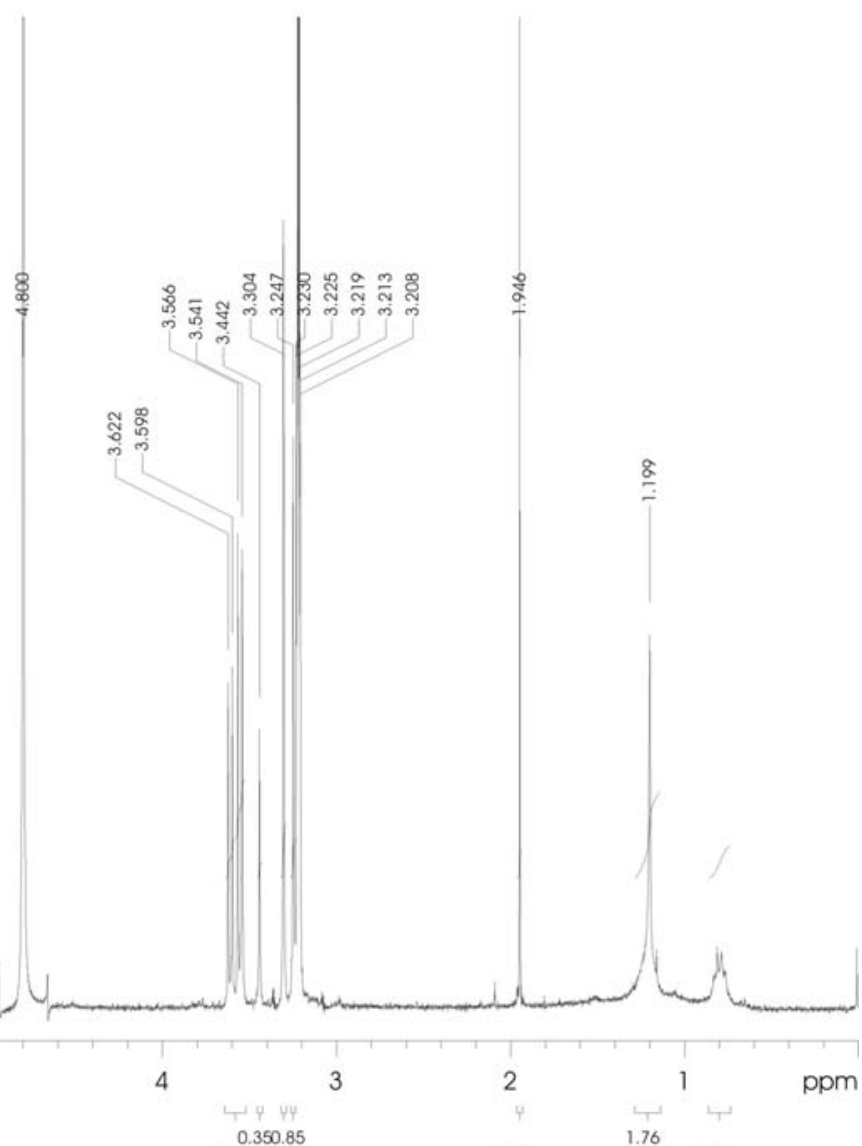

200111

0.59

0.67

Solvent: CD3OD

Amblent temperatu GEMINI-300BB "pss116"

Relax. delay 1.000 sec
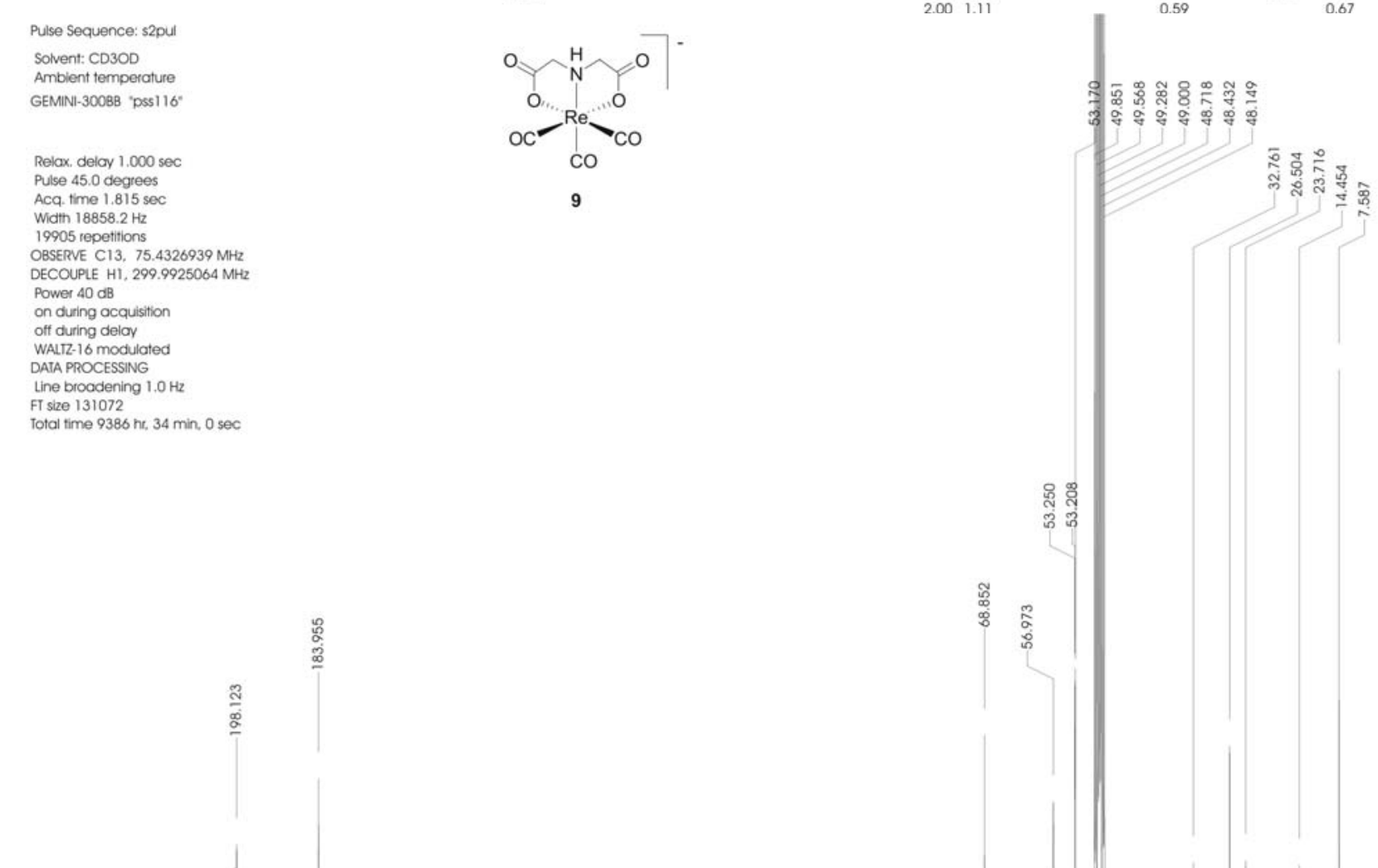
NMR spectra of complex 6:
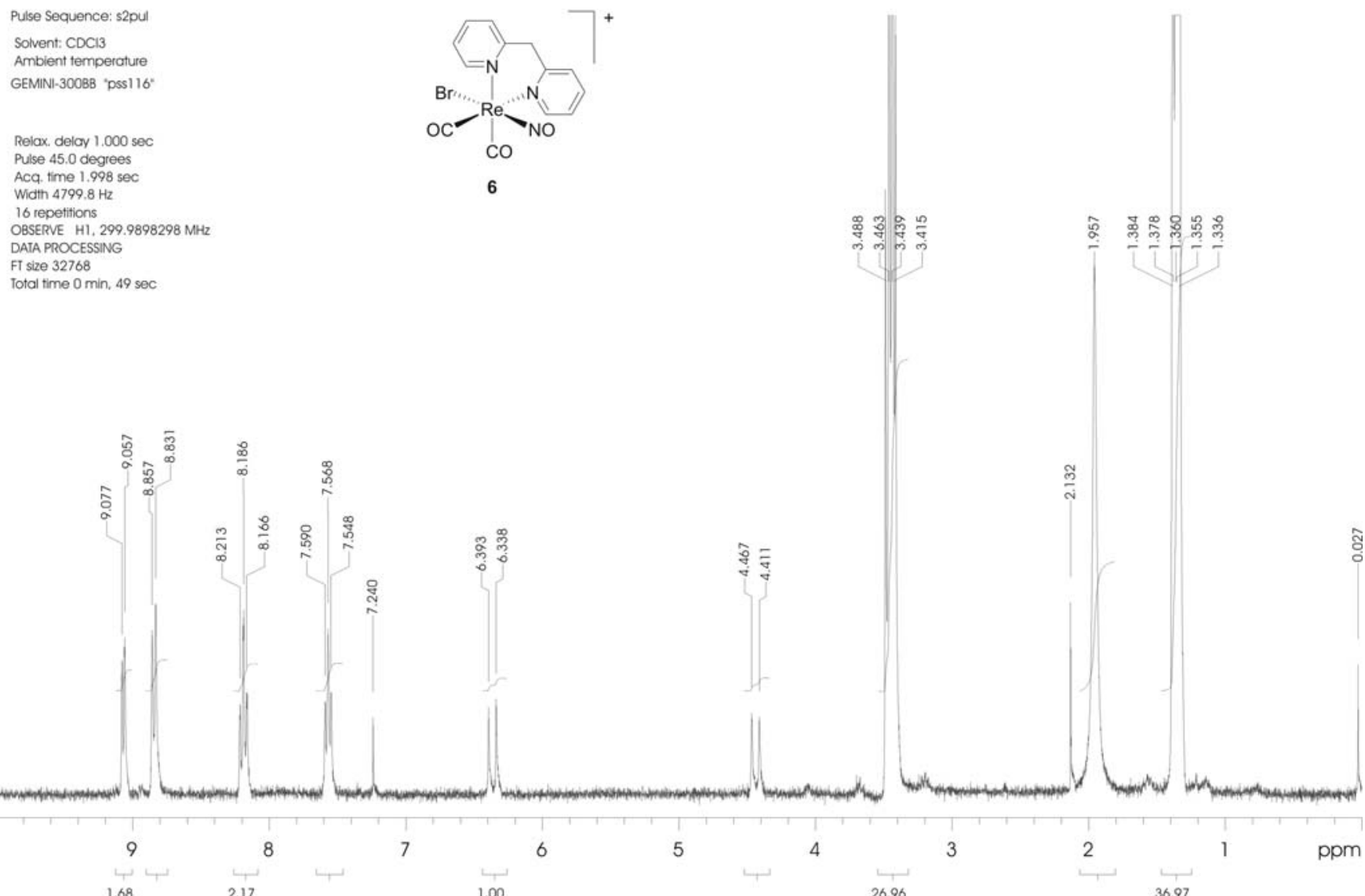

Pulse Sequence: 22 put

2.22

Solvent: $\mathrm{CDCl}^{3}$

Temp. $23.0 \mathrm{C} / 296.1 \mathrm{~K}$

GEMINI-3008B "pss116"

Relax. delay $1.000 \mathrm{sec}$

Pulse 45.0 degrees

Acq. fime $1.815 \mathrm{sec}$

1800 repetitions

OBSERVE C13. $75.4324966 \mathrm{MHz}$

DECOUPLE H1, $299.9913244 \mathrm{MHz}$

Power $40 \mathrm{~dB}$

on during acquisition

off during delay

WALIZ-16 modulated

DATA PROCESSING

Line broadening $1.0 \mathrm{H}$

FT size 131072

Total fime $93 \mathrm{hr}, 51 \mathrm{~min}, 57 \mathrm{sec}$

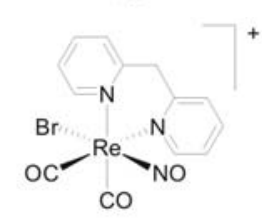

6

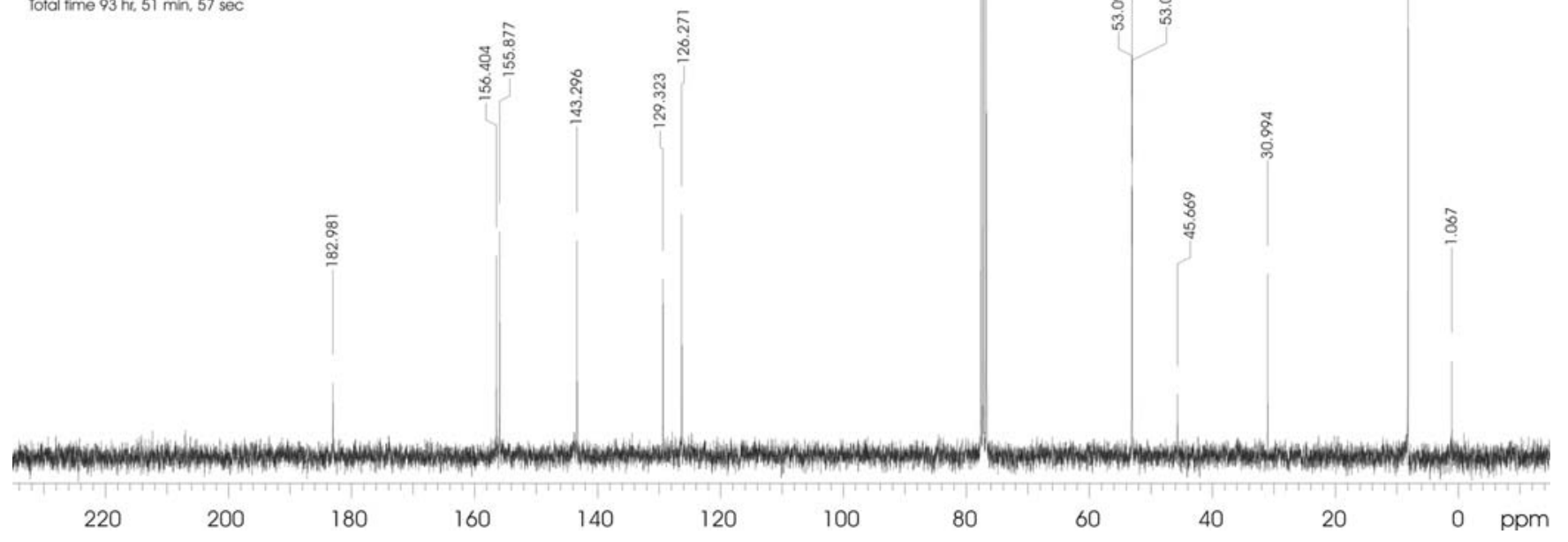


NMR spectra of complex 10:

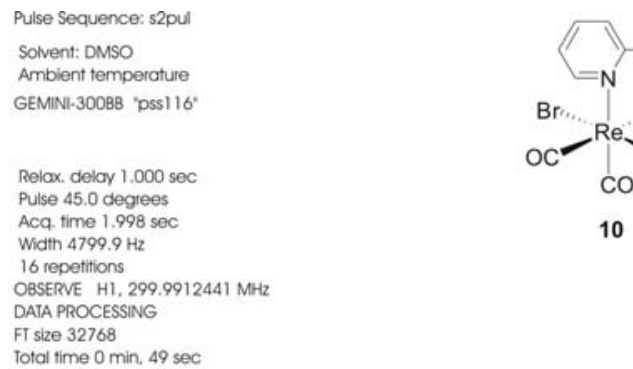

10

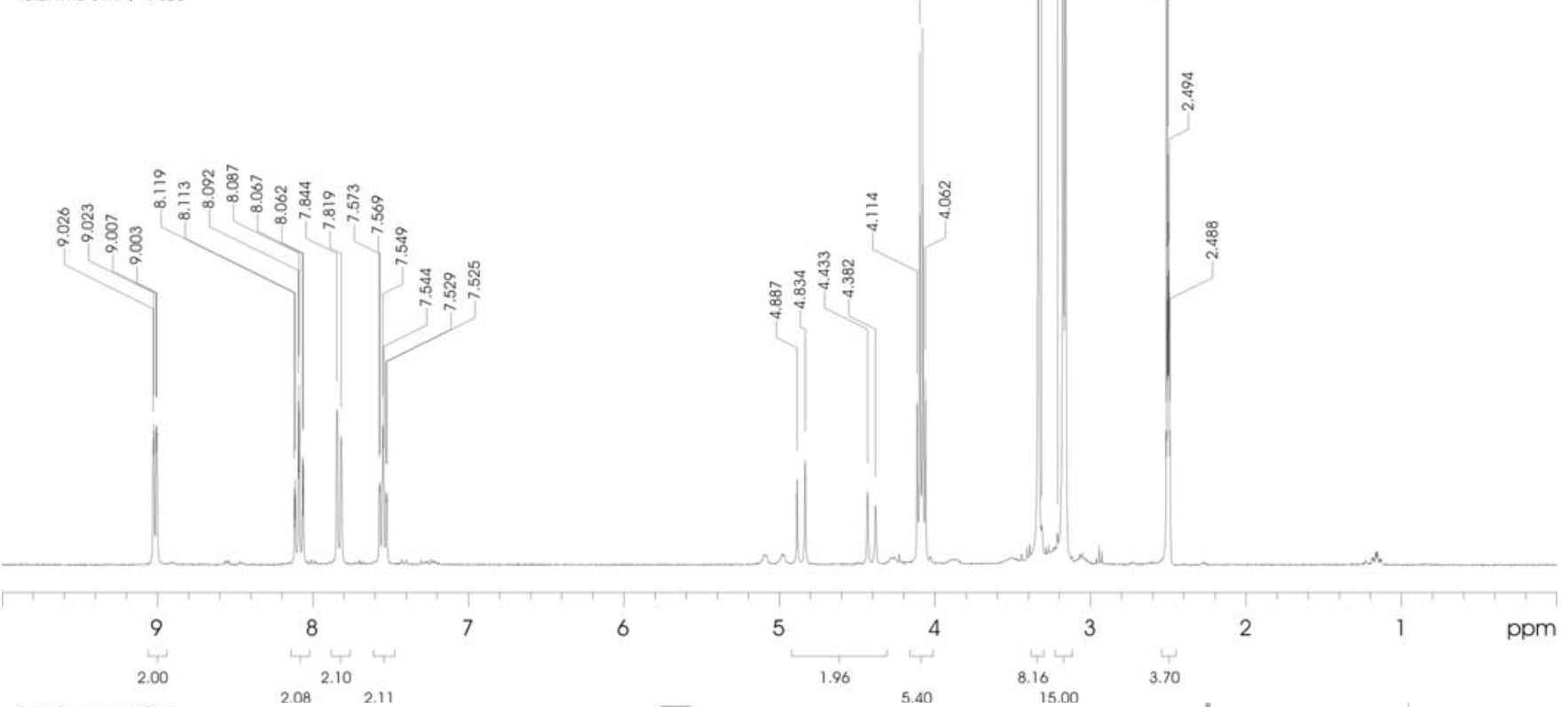

Pulse Sequence: s2pul

Solvent: DMSO

Temp. $23.0 \mathrm{C} / 296.1 \mathrm{~K}$

GEMINI-300BB 'pSS116"

Relax, delay $1.000 \mathrm{sec}$

Pulse 45.0 degrees

Acq. time $1.815 \mathrm{sec}$

Width $18858.2 \mathrm{~Hz}$

OBSERVE C13, 75.4328977 MH2

DECOUPIE H1. $299.9927494 \mathrm{MHz}$

Power $40 \mathrm{~dB}$

on during acquistion

off during delay

WALIZ-16 modulated

DAIA PROCESSING

ine broadening 1.0

FT size 131072

Total time $8 \mathrm{he}, 51 \mathrm{~min}, 16 \mathrm{sec}$

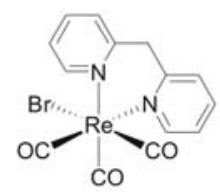

10

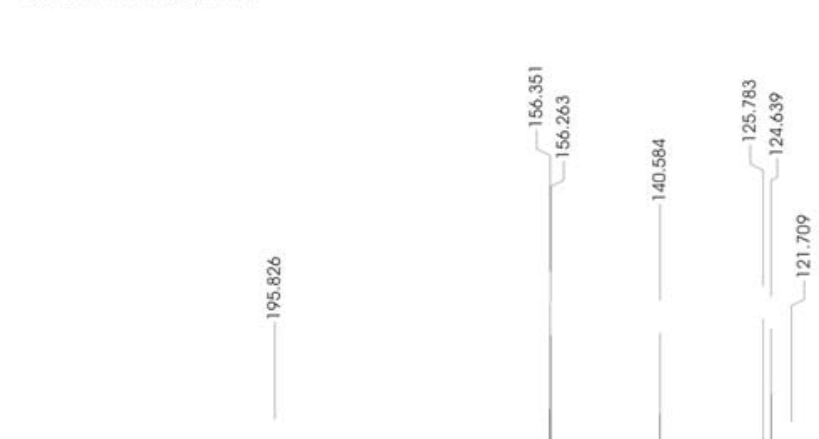

